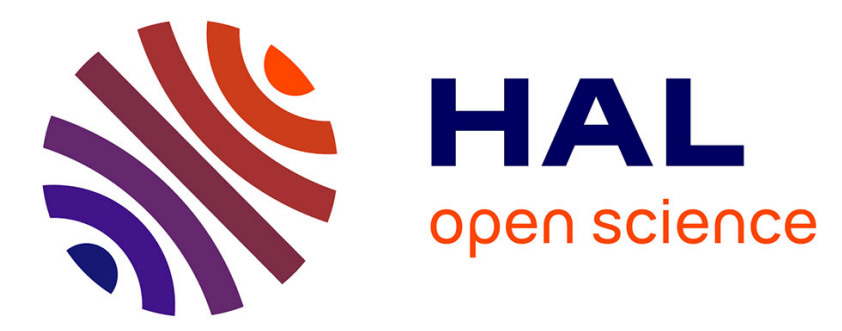

\title{
On the unknown input observer design : a decoupling class approach
}

\author{
Souad Bezzaoucha, Benoît Marx, Didier Maquin, José Ragot
}

\section{To cite this version:}

Souad Bezzaoucha, Benoît Marx, Didier Maquin, José Ragot. On the unknown input observer design : a decoupling class approach. 9th IEEE International Conference on Control \& Automation, ICCA'11, Dec 2011, Santiago, Chile. pp.602-607, 10.1109/ICCA.2011.6138035 . hal-00630362

\section{HAL Id: hal-00630362 https://hal.science/hal-00630362}

Submitted on 8 Apr 2014

HAL is a multi-disciplinary open access archive for the deposit and dissemination of scientific research documents, whether they are published or not. The documents may come from teaching and research institutions in France or abroad, or from public or private research centers.
L'archive ouverte pluridisciplinaire HAL, est destinée au dépôt et à la diffusion de documents scientifiques de niveau recherche, publiés ou non, émanant des établissements d'enseignement et de recherche français ou étrangers, des laboratoires publics ou privés. 


\title{
On the Unknown Input Observer Design : a Decoupling Class Approach
}

\author{
Souad Bezzaoucha, Benoît Marx, Didier Maquin, José Ragot
}

\begin{abstract}
This paper deals with state estimation for linear discrete-time systems subject to unknown input. Although many papers have dealt with the problem of Unknown Input Observer design, state decoupling and reconstruction; the goal is to present a new method allowing to characterize a class of unknown inputs to which the estimation error is decoupled. This contribution considers two problems : exact decoupling and $\mathscr{L}_{2}$-attenuation of the unknown input to the state estimation error.
\end{abstract}

\section{INTRODUCTION}

The Unknown Input Observer (UIO) design has received considerable interest due to its importance and connection with fault detection problem, since in many cases a part of the system's input is inaccessible (e.g. plant disturbance or actuator failure). Under such circumstances, a conventional observer that requires knowledge of all inputs cannot be used directly, then the UIO was developed to estimate the state of an uncertain system despite the existance of unknown inputs or uncertain disturbances [4], [2], [7], [9], [11], [12].

Several researches were achieved concerning the state estimation in the presence of unknown inputs. They can be gathered into two categories. The first one supposes an a priori knowledge on these nonmeasurable inputs; in particular, Johnson [16] proposes a polynomial approach and Hostetter and Meditch [11] suggest approximating the unknown inputs by the response of a known dynamic system [1].

The second category proceeds either by estimation of the unknown inputs [17] or by their complete elimination from the system equation. However, some of these methods require differentiation of the measured outputs which can amplify the effect of the measurement's noise.

One of the most successful robust observer design methods ressorts to the disturbance decoupling principle [25] [12] [7]. The problem of UIO has been initialised by Basile and Marro [2], Guidorzi and Marro [9]. Since then, several contributions for UIOs have been proposed [4], [10]. For these methods, a rank condition relating the output distribution matrix and the input distribution matrices must be satisfied which is sometimes difficult and might be quite restrictive.

An approach to simultaneously estimate the unknown input and the system state using the PI observer has been

The authors are with Centre de Recherche en Automatique de Nancy (CRAN), Nancy-University, CNRS, 2 avenue de la Forêt de Haye F-54516 Vandoeuvre-les-Nancy. firstname.namedensem.inpl-nancy.fr proposed by [23], [13] and [14]. However, this observer concerns the case of constant unknown inputs. In [18], a model-based oserver design in the presence of polynomial unknown inputs for TS fuzzy systems has been investigated. In [20], the authors present a method for state-estimation for Takagi-Sugeno descriptor systems affected by UI. Sufficient existence conditions of the unknown inputs decoupling observers are given and strict linear matrix inequalities are solved to determine the gain of the observers so that the estimated state asymptotically tends to the real one.

Summarizing, the UIO design is based either on the decoupling such that the estimation error must not depend on the the UI, or on the synthesis of an Integral Observer for the detection (estimation) of disturbances. But, as much as the authors know, all the proposed strategies impose structural and rank constraints.

This work advantage is to propose an exact decoupling instead of an attenuation or a decoupling for a constant UI. Moreover, it is proposed to decompose any UI into two terms. The first one is a sum of exponential functions from which the state estimates can be exactly decoupled. For a given system, the class of the UI satisfying that property is clearly satisfied. The effect of the remaining part of the UI on the state estimates is then attenuated in an $\mathscr{L}_{2}$ framework. Two cases will be treated, the exact decoupling case and the almost-exact case which consists in decoupling the estimation to a subset of the UI, while attenuing the $\mathscr{L}_{2}$ gain from the other UI to the estimation.

This paper is organised as follows : Section II presents a second order system in order to introduce the decoupling strategy and the Uknown Input Class for exact decoupling notion and how to generate this class. Section III is a generalization of the second section. In section IV, we introduce the notion of partial decoupling and the linear matrix inequalities conditions to ensure the $\mathscr{L}_{2}$ attenuation of the UI effect on the system. In order to impove the obtained results, a pole placement will also be applied.

However, the usual linearization approaches are not suitable to the present problem since BMIs (Bilinear Matrix Inequalities) are to be dealt with. A gain adjustment technique is then applied. This synthesis linearize the inequalities by fixing one of the unknown variable [19]. This kind of procedure can be found in the centrage-XY procedure [15], the D-K iteration mentionned in [22] or Yamada's approach [24]. However, there is no guarantee that the proposed structure assures the algorithm convergence.

Finally, in the last section, simulations are presented to show 
the efficiency of the proposed approach.

\section{ILlustrative EXAMPLE}

To begin with, the procedure is introduced with the help of a simple example being a second order system. The different steps leading to the Uknown Input Class for exact decoupling are detailed. Consider a second order system described by :

$$
\left\{\begin{array}{l}
x_{k+1}=A x_{k}+B u_{k}+D \eta_{k-1} \\
y_{k}=C x_{k}+e \eta_{k-1}
\end{array}\right.
$$

Vectors $x_{k} \in \mathbb{R}^{2}, u_{k} \in \mathbb{R}, \eta_{k} \in \mathbb{R}$ and $y_{k} \in \mathbb{R}$ are the system state, input, unknown input and the output vector respectively. The system matrices are real valued, constant and of appropriate dimensions :

$$
\begin{gathered}
x_{k}=\left(\begin{array}{c}
x_{1 k} \\
x_{2 k}
\end{array}\right) \quad A=\left(\begin{array}{ll}
a_{11} & a_{12} \\
a_{21} & a_{22}
\end{array}\right) \quad B=\left(\begin{array}{l}
b_{1} \\
b_{2}
\end{array}\right) \\
C=\left(\begin{array}{ll}
c_{1} & c_{2}
\end{array}\right) \quad D=\left(\begin{array}{l}
d_{1} \\
d_{2}
\end{array}\right)
\end{gathered}
$$

The proposed Proportional Integral Observer (PIO) of gain $K$ and the UIO depending on an auxiliary variable $z_{k} \in \mathbb{R}$ are respectivelly given by the following equations :

$$
\begin{gathered}
\left\{\begin{array}{l}
\hat{x}_{k+1}=A \hat{x}_{k}+B u_{k}+D \hat{\eta}_{k-1}+K \tilde{y}_{k} \\
\hat{y}_{k}=C \hat{x}_{k}+e \hat{\eta}_{k-1} \\
\tilde{y}_{k}=y_{k}-\hat{y}_{k}
\end{array}\right. \\
\left\{\begin{array}{l}
z_{k+1}=\gamma_{1} \tilde{y}_{k}+\lambda_{1} z_{k} \\
\hat{\eta}_{k+1}=\gamma_{2} z_{k}+\lambda_{2} \hat{\eta}_{k}
\end{array}\right.
\end{gathered}
$$

with :

$$
K=\left(\begin{array}{l}
k_{1} \\
k_{2}
\end{array}\right)
$$

It can be noted that the UIO has a filter structure with as an input the output reconstruction error $\tilde{y}_{k}$. This filter parameters $\gamma_{1}, \gamma_{2}, \lambda_{1}$ and $\lambda_{2}$ allow to modify the gain and time constant of the UIO.

In (4), only one integrator is used but this structure can be generalized to multiple integrator observer. However, the choice $z_{k+1}=\gamma_{1} \tilde{y}_{k+1}+\lambda_{1} z_{k}$ with $\gamma_{1}=1$ and $\lambda_{1}=0$ leads to $z_{k+1}=\tilde{y}_{k}$ which is equivalent to a first order filter for the UI estimation. The system observer is described by equations (3) and (4). The gains $K, \gamma_{1}, \gamma_{2}, \lambda_{1}$ and $\lambda_{2}$ are choosen according to the state and UI reconstruction specifications. In the following, the state and UI estimation errors are expressed as function of the UI. Since the system and its observer are linear, the time operator $q$, is adequate to express the errors :

$$
\begin{aligned}
& \tilde{x}_{k}=x_{k}-\hat{x}_{k} \\
& \tilde{\eta}_{k}=\eta_{k}-\hat{\eta}_{k}
\end{aligned}
$$

From equations (1), (3) and (4), the state reconstruction error function of the UI is given by :

$$
\left\{\begin{array}{l}
\tilde{x}_{1 k}=\frac{N_{1}(q)}{D(q)} \eta_{k} \\
\tilde{x}_{2 k}=\frac{N_{2}(q)}{D(q)} \eta_{k}
\end{array}\right.
$$

$$
\left\{\begin{array}{l}
N_{1}(q)=\left(q-\lambda_{1}\right)\left(q-\lambda_{2}\right)\left(\tilde{a}_{12} \tilde{d}_{2}-\tilde{a}_{22} \tilde{d}_{1}+q \tilde{d}_{1}\right) \\
N_{2}(q)=\left(q-\lambda_{1}\right)\left(q-\lambda_{2}\right)\left(\tilde{a}_{21} \tilde{d}_{1}-\tilde{a}_{11} \tilde{d}_{2}+q \tilde{d}_{2}\right) \\
D(q)=\left(\left(q-\lambda_{1}\right)\left(q-\lambda_{2}\right)+\gamma e\right)\left(\left(q-\tilde{a}_{11}\right)\left(q-\tilde{a}_{22}\right)-\tilde{a}_{12} \tilde{a}_{21}\right) \\
+\gamma c_{1}\left(\tilde{a}_{12} \tilde{d}_{2}-\tilde{a}_{22} \tilde{d}_{1}+q \tilde{d}_{1}\right)+\gamma c_{2}\left(\tilde{a}_{21} \tilde{d}_{1}-\tilde{a}_{11} \tilde{d}_{2}+q \tilde{d}_{2}\right)
\end{array}\right.
$$

with :

$$
\left\{\begin{array}{l}
\tilde{a}_{11}=a_{11}-k_{1} c_{1} \\
\tilde{a}_{12}=a_{12}-k_{1} c_{2} \\
\tilde{a}_{21}=a_{21}-k_{2} c_{1} \\
\tilde{a}_{22}=a_{22}-k_{2} c_{2} \\
\tilde{d}_{1}=d_{1}-k_{1} e \\
\tilde{d}_{2}=d_{2}-k_{2} e
\end{array}\right.
$$

From (5), conditions for the estimation errors to be independent from the UI can easily be derived. Then, the UI familly satisfying an exact decoupling is solution of :

$$
\left\{\begin{array}{l}
\frac{N_{1}(q)}{D(q)} \eta_{k}=0 \\
\frac{N_{2}(q)}{D(q)} \eta_{k}=0
\end{array}\right.
$$

In order to find the solution $\eta_{k}$ assuring the precedent condition, it is imposed that $N_{1}(q)$ and $N_{2}(q)$ have the same roots. It should also be checked if some solutions are common to $D(q)$ and $N_{1}(q)$ (or $N_{2}(q)$ ). For the first point, we have the following condition :

$$
\frac{\tilde{a}_{11} \tilde{d}_{2}-\tilde{a}_{22} \tilde{d}_{1}}{\tilde{a}_{21} \tilde{d}_{1}-\tilde{a}_{11} \tilde{d}_{2}}=\frac{\tilde{d}_{1}}{\tilde{d}_{2}}
$$

which imposes a constraint on the observer coefficients $k_{1}$ and $k_{2}$. That leads to :

$$
q_{0}=\frac{\tilde{a}_{21} \tilde{d}_{1}-\tilde{a}_{11} \tilde{d}_{2}}{\tilde{d}_{2}}
$$

is a common root between $N_{1}(q), N_{2}(q)$ and $D(q)$.

Then (7) is written as :

$$
\left(q-\lambda_{1}\right)\left(q-\lambda_{2}\right) \eta_{k}=0
$$

The solution is given by an UI being the sum of two exponential functions :

$$
\eta_{k}=A_{1} \lambda_{1}^{k}+A_{2} \lambda_{2}^{k}
$$

where coefficients $A_{1}$ and $A_{2}$ are arbitrarily set. Finally, the choice of the observer values $\lambda_{1}$ and $\lambda_{2}$ gives the UI class assuring the exact decoupling of the state error toward the UIO for any values of the coefficients $A_{1}$ and $A_{2}$.

\section{RECONSTRUCTION ERRORS : DISTURBANCES DECOUPLING}

Let us define the following system equations :

$$
\left\{\begin{array}{l}
x_{k+1}=A x_{k}+B u_{k}+D \eta_{k-1} \\
y_{k}=C x_{k}+e \eta_{k-1}
\end{array}\right.
$$

Vectors $x_{k} \in \mathbb{R}^{n}, u_{k} \in \mathbb{R}^{m}, \eta_{k} \in \mathbb{R}$ and $y_{k} \in \mathbb{R}^{p}$ are the system state, input, unknown input and the output vectors respectively. The system matrices $A \in \mathbb{R}^{n \times n}, B \in \mathbb{R}^{n \times m}, D \in$ $\mathbb{R}^{n \times 1}, C \in \mathbb{R}^{p \times n}$ et $e \in \mathbb{R}^{p \times 1}$ are real valued and known. 
The proposed system observer of gain $K$ and the UIO are respectivelly given by the following equations :

$$
\begin{gathered}
\left\{\begin{array}{l}
\hat{x}_{k+1}=A \hat{x}_{k}+B u_{k}+D \hat{\eta}_{k-1}+K \tilde{y}_{k} \\
\hat{y}_{k}=C \hat{x}_{k}+e \hat{\eta}_{k-1} \\
\tilde{y}_{k}=y_{k}-\hat{y}_{k}
\end{array}\right. \\
\left\{\begin{array}{l}
z_{k+1}=\Gamma \tilde{y}_{k}+\Lambda z_{k} \\
\hat{\eta}_{k+1}=\gamma z_{k}+\lambda \hat{\eta}_{k}
\end{array}\right.
\end{gathered}
$$

with appropriate dimensions $: z_{k} \in \mathbb{R}^{q}, K \in \mathbb{R}^{n \times p}, \Gamma \in \mathbb{R}^{q \times p}$, $\gamma \in \mathbb{R}^{1 \times q}, \Lambda \in \mathbb{R}^{q \times q}$ and $\lambda \in \mathbb{R}$.

Depending on the value of the UIO parameters, we can have either a proportional observer, an integral or a multiple integral observer. In this particular case, the choice $\lambda_{1}=1$ or $\lambda_{2}=1$ introduces the two integrators in this filter structure. By following the same steps as in the previous section, the state and UI reconstruction errors are expressed; we get from equation (14) with the time operator $q$ :

$$
\begin{gathered}
\hat{\eta}_{k}=(q-\lambda)^{-1} \gamma z_{k} \\
\left(q I_{q}-\Lambda\right) z_{k}=\Gamma \tilde{y}_{k}
\end{gathered}
$$

From (15), (16), (12) and (13), we have :

$$
\left(q I_{q}-\Lambda\right) z_{k}=\Gamma C \tilde{x}_{k}+\Gamma e\left(\eta_{k-1}-\hat{\eta}_{k-1}\right)
$$

which leads to :

$$
\left[\left(q I_{q}-\Lambda\right)+\Gamma e(q-\lambda)^{-1} q^{-1} \gamma\right] z_{k}=\Gamma C \tilde{x}_{k}+\Gamma e q^{-1} \eta_{k}
$$

The state error $\tilde{x}_{k}$ dynamic is obtained from (12) and (13) :

$$
\begin{array}{ll}
\tilde{x}_{k+1} & =\bar{A} \tilde{x}_{k}+\bar{D} \tilde{\eta}_{k-1} \\
\bar{A} & =A-K C \\
\bar{D} & =D-K e
\end{array}
$$

From (18) and (16), we have :

$$
\begin{aligned}
\left(q I_{n}-\bar{A}\right) \tilde{x}_{k} & =\bar{D} \tilde{\eta}_{k-1} \\
& =\bar{D} q^{-1} \eta_{k}-\bar{D} q^{-1}(q-\lambda)^{-1} \gamma z_{k}
\end{aligned}
$$

That gives the state estimation error :

$$
\tilde{x}_{k}=\left(q I_{n}-\bar{A}\right)^{-1} \bar{D} q^{-1} \eta_{k}-\left(q I_{n}-\bar{A}\right)^{-1} \bar{D} q^{-1}(q-\lambda)^{-1} \gamma_{z_{k}}
$$

By replacing this expression in (17), we have :

$$
z_{k}=\bar{Z}^{-1} \bar{\Lambda} \eta_{k}
$$

with :

$$
\begin{aligned}
& \bar{\Lambda}=\Gamma C\left(q I_{n}-\bar{A}\right)^{-1} \bar{D}+\Gamma e \\
& \bar{Z}=q\left(q I_{q}-\Lambda\right)+\bar{\Lambda}(q-\lambda)^{-1} \gamma
\end{aligned}
$$

Finally, replacing (20) in (15) and (19) to have :

$$
\left\{\begin{array}{l}
\hat{\eta}_{k}=(q-\lambda)^{-1} \gamma \bar{Z}^{-1} \bar{\Lambda} \eta_{k} \\
\tilde{x}_{k}=\left(q I_{n}-\bar{A}\right)^{-1} \bar{D} q^{-1}\left[1-(q-\lambda)^{-1} \gamma \bar{Z}^{-1} \bar{\Lambda}\right] \eta_{k}
\end{array}\right.
$$

the UI estimation error becomes :

$$
\tilde{\eta}_{k}=\left[1-(q-\lambda)^{-1} \gamma \bar{Z}^{-1} \bar{\Lambda}\right] \eta_{k}
$$

From (22) we have the state estimation error decoupling condition toward the UI :

$$
\left(q I_{n}-\bar{A}\right)^{-1} \bar{D} q^{-1}\left[1-(q-\lambda)^{-1} \gamma \bar{Z}^{-1} \bar{\Lambda}\right] \eta_{k}=0
$$

In order to decouple the state from the UI and assure an exact estimation of it, the following condition has to be verified :

$$
\left[1-(q-\lambda)^{-1} \gamma \bar{Z}^{-1} \bar{\Lambda}\right] \eta_{k}=0
$$

Equation (24) may be extended as $\frac{N(q)}{D(q)} \eta_{k}=0$. From solving this last equation, we have the roots that define the UI class that ensure an exact decoupling of the estimation error toward the UI. This class is written as : $\sum_{i} A_{i} \lambda_{i}$ where the $\lambda_{i}$ correspond to the roots of (24) and $A_{i}$ are totally free parameters.

\section{Partial Decoupling Observer}

In the previous section, was detailed how to find the class of UI ensuring an exact decoupling of the UI in respect to the state estimation error. In the following section, a general case with an UI that does not respond to the decoupling condition is considered. In this case, the problem is solved by attenuating the effect (transfer) of the UI to the estimation error and propose linear matrix inequalities to determinate the observer gain so that the estimated state asymptotically tends to the real one.

In addition to the two previous cases (exact and partial decoupling), we also have a third one, which is a mixt between the two solutions. In fact, any UI may be decomposed into a sum of two terms $\eta_{k}=\eta_{k}^{d}+\eta_{k}^{a}$. The first term corresponds to the decoupling term and the second one is the approximaion term onto $\mathscr{L}_{2}$ attenuation is applied. In subsection $A$, we only present the attenuation approach; but, in the simulation section the combined approach will be illustrated.

\section{A. $\mathscr{L}_{2}$ Attenuation}

System and observer equations are given by :

$$
\left\{\begin{array}{l}
\tilde{x}_{k+1}=\bar{A} \tilde{x}_{k}+\bar{D} \tilde{\eta}_{k-1} \\
\tilde{\eta}_{k}=\eta_{k}-\lambda \eta_{k-1}-\gamma z_{k-1}+\lambda \tilde{\eta}_{k-1} \\
z_{k+1}=\Gamma C \tilde{x}_{k}+\Gamma e \tilde{\eta}_{k-1}+\Lambda z_{k}
\end{array}\right.
$$

The corresponding matrix form is given by :

$$
e_{k+1}=A_{1} e_{k}+B_{1} \eta_{k}^{a}
$$

with :

$$
\begin{gathered}
A_{1}=\left(\begin{array}{cccc}
\bar{A} & \bar{D} & 0 & 0 \\
0 & \lambda & 0 & -\gamma \\
\Gamma C & \Gamma e & \Lambda & 0 \\
0 & 0 & 1 & 0
\end{array}\right), B_{1}=\left(\begin{array}{cc}
0 & 0 \\
1-\lambda \\
0 & 0 \\
0 & 0
\end{array}\right), \\
e_{k}=\left(\begin{array}{c}
\tilde{x}_{k} \\
\tilde{\eta}_{k-1} \\
z_{k} \\
z_{k-1}
\end{array}\right), \eta_{k}^{a}=\left(\begin{array}{c}
\eta_{k} \\
\eta_{k-1}
\end{array}\right)
\end{gathered}
$$

In particular, (26) gives the UI influence on the estimation errors. To focus on the impact of the UI on the state estimation, a new observer output is considered :

$$
g_{k}=C_{1} e_{k}
$$

with : $C_{1}=\left(\begin{array}{llll}I & 0 & 0 & 0\end{array}\right)$.

Considering the Real Bounded Lemma [3], the system (26), 
it is stable and the $\mathscr{L}_{2}$ gain is bounded by $\frac{\left\|g_{k}\right\|_{2}}{\left\|\eta_{k}\right\|_{2}}<\mu$ if there exists a positive symmetric matrice $P$ and a positive scalar $\mu$ such that the following condition holds :

$$
\left[\begin{array}{ccc}
A_{1}^{T} P A_{1}-P & A_{1}^{T} P B_{1} & C_{1}^{T} \\
B_{1}^{T} P A_{1} & B_{1}^{T} P B_{1}-\mu^{2} I & 0 \\
C_{1} & 0 & -\mu^{2} I
\end{array}\right]<0
$$

According to [8] and [21], the previous problem can be reformulated by searching a positive symetric definite matrice $P$, gains $K$ and $G$ such that :

$$
\left[\begin{array}{cccc}
-P & A_{1}^{T} P B_{1} & C_{1}^{T} & A_{1}^{T} G^{T} \\
B_{1}^{T} P A_{1} & B_{1}^{T} P B_{1}-\mu^{2} I & 0 & 0 \\
C_{1} & 0 & -\mu^{2} I & 0 \\
G A_{1} & 0 & 0 & -G-G^{T}+P
\end{array}\right]<0
$$

where $A_{1}$ defined in (28) with the help of (19), depends on $K$. Let us remark that inequality (31) is not linear. For that reason some transformations are needed to obtain LMIs. Let us write the matrix $A_{1}$ such that :

$$
A_{1}=\bar{A}_{1}-R K \bar{B}_{1}
$$

with :

$\bar{A}_{1}=\left(\begin{array}{cccc}A & D & 0 & 0 \\ 0 & \lambda & 0 & -\gamma \\ \Gamma C & \Gamma e & \Lambda & 0 \\ 0 & 0 & 1 & 0\end{array}\right) R=\left(\begin{array}{l}I \\ 0 \\ 0 \\ 0\end{array}\right) \bar{B}_{1}=\left(\begin{array}{llll}C & e & 0 & 0\end{array}\right)$

Replacing $A_{1}$ by (31) in (30), we have :

$$
\left[\begin{array}{cccc}
-P & \bar{A}_{1}^{T} P B_{1} & C_{1}^{T} & \bar{A}_{1}^{T} G^{T} \\
B_{1}^{T} P \bar{A}_{1} & B_{1}^{T} P B_{1}-\bar{\mu} I & 0 & 0 \\
C_{1} & 0 & -\bar{\mu} I & 0 \\
G \bar{A}_{1} & 0 & 0 & P-G-G^{T}
\end{array}\right]+M^{T} N+N^{T} M<0
$$

with $M=\left[\begin{array}{c}-\bar{B}_{1}^{T} K^{T} \\ 0 \\ 0 \\ 0\end{array}\right]^{T}, N=\left[\begin{array}{c}0 \\ B_{1}^{T} P R \\ 0 \\ G R\end{array}\right]^{T}$ and $\bar{\mu}=\mu^{2}$

Let us recall the following lemma [26]. Consider two real matrices $\Pi$ and $\Lambda$ with appropriate dimensions, for any positive matrix $\Sigma$ the following inequality holds :

$$
\Pi^{T} \Lambda+\Lambda^{T} \Pi \leq \Pi^{T} \Sigma \Pi+\Lambda^{T} \Sigma^{-1} \Lambda
$$

Applying this lemma, (33) becomes :

$$
\left[\begin{array}{cccc}
-P & \bar{A}_{1}^{T} P B_{1} & C_{1}^{T} & \bar{A}_{1}^{T} G^{T} \\
B_{1}^{T} P \bar{A}_{1} & B_{1}^{T} P B_{1}-\bar{\mu} I & 0 & 0 \\
C_{1} & 0 & -\bar{\mu} I & 0 \\
G \bar{A}_{1} & 0 & 0 & P-G-G^{T}
\end{array}\right]++
$$

Applying Schur's complement, we get :

$$
\left[\begin{array}{cccccc}
-P & \bar{A}_{1}^{T} P B_{1} & C_{1}^{T} & \bar{A}_{1}^{T} G^{T} & \bar{B}_{1}^{T} K^{T} & 0 \\
B_{1}^{T} P & B_{1}^{T} P B_{1}-\bar{\mu} I & 0 & 0 & 0 & B_{1}^{T} P R \\
C_{1} & 0 & -\bar{\mu} & 0 & 0 & 0 \\
G \bar{A}_{1} & 0 & 0 & -G-G^{T}+P & 0 & G R \\
K \bar{B}_{1} & 0 & 0 & 0 & -\Sigma^{-1} & 0 \\
0 & R^{T} P B_{1} & 0 & R^{T} G^{T} & 0 & -\Sigma
\end{array}\right]_{(36)}
$$

At last, (36) becomes :

$$
\left[\begin{array}{cccccc}
-P & \bar{A}_{1}^{T} P B_{1} & C_{1}^{T} & \bar{A}_{1}^{T} G^{T} & \bar{B}_{1}^{T} F^{T} & 0 \\
B_{1}^{T} P & B_{1}^{T} P B_{1}-\bar{\mu} I & 0 & 0 & 0 & B_{1}^{T} P R \\
C_{1} & 0 & -\bar{\mu} & 0 & 0 & 0 \\
G \bar{A}_{1} & 0 & 0 & -G-G^{T}+P & 0 & G R \\
F \bar{B}_{1} & 0 & 0 & 0 & -\Sigma^{T} & 0 \\
0 & R^{T} P B_{1} & 0 & R^{T} G^{T} & 0 & -\Sigma
\end{array}\right]<0
$$

with $F=\Sigma K$. The LMI must be solved in $P, G, F$ and the gain $K$ is obtained by $K=\Sigma^{-1} F$.

\section{B. Pole Assignment}

The minimization of the attenuation factor $\mu$ may result in slow dynamics of the state estimation error. This problem can be solved by pole assignment of the closed loop system in a specified region. The considered region is a disk centred at $(q, 0)$ with radius $\alpha$. Thus, the condition to answer this constraint is given by the following : find $P=P^{T}>0$ and $Q=Q^{T}>0$ such that the following LMI [6] occurs :

$$
\left[\begin{array}{cc}
-\alpha Q & -q Q+Q A-G C \\
(-q Q+Q A-G C)^{T} & -\alpha P
\end{array}\right]<0
$$

with $G=Q K$. We have to solve this LMI regarding to $Q$ and $G$ then we deduce $K$. Thus, to ensure the stabilty and pole assignment, the conditions (37) and (38) must be fulfilled simultaneously.

\section{Gain Ajustement}

From matrices $F$ and $G$ definitions, there is a dependence between the two LMIS (37) and (38). Then we have to solve simultaneously these two conditions. It can be noted $L M I 1(P, K)$ and $L M I 2(Q, K)$. The proposed method is based on an ajustment technique allowing to set some variables and calculate others in an iterative way. More precisely, if the gain $K$ is fixed, we solve $L M I 1(P, K)$ regarding to $P$. Then we solve $L M I 2(Q, K)$ regarding to $Q$ and $K$ and we use the obtained result $K$ for another cycle (see table 1). This procedure was choosen in reason of its simplicity, but one should be aware that no optimality or convergence guarantee is given. However, since our study goal is to find a solution to the given conditions, an optimal solution is not a necessity.

Iterative optimisation for gain $K$ :

1) Set $i=0$. Chose a stabilisable value $K_{0}$. Put $K^{(i)}=K_{0}$.

2) $\mathscr{L}_{2}$ attenuation : Find $P^{(i+1)}>0$ solution of $\operatorname{LMI} 1\left(P, K^{(i)}\right)$.

3) Pole assignment : Find $Q^{(i+1)}$ and $K^{(i+1)}$ solution of $L M I 2(Q, K)$.

4) Stopping condition :

- If $\left\|K^{(i+1)}-K^{(i)}\right\|<\varepsilon$ stop the algorithm : $K_{\text {final }}=$ $K^{(i+1)}$.

- Else, set $i=i+1$ and go back to step 2 .

Table1 : Adjustment algorithm 


\section{Simulations}

Consider the following system described by :

$$
\begin{array}{r}
A=\left(\begin{array}{cccc}
0.6 & -0.2 & -0.1 & 0.1 \\
-0.1 & 0.7 & -0.1 & 0.1 \\
0.4 & 0 & 0.9 & -0.1 \\
0 & 0.2 & 0 & 0.8
\end{array}\right) \quad B=\left(\begin{array}{cc}
-0.3 & -0.4 \\
0.5 & -0.4 \\
-0.1 & 0.6 \\
-0.2 & 0.7
\end{array}\right) \\
C=\left(\begin{array}{lll}
1 & 1 & 1
\end{array}\right) \quad D=\left(\begin{array}{c}
0.2 \\
-0.3 \\
0.1 \\
0.1
\end{array}\right) \quad E=-4.5
\end{array}
$$

with the observer parameters :

$$
\Lambda=1 ; \lambda=0.7 \text { et } \Gamma=0.2 ; \gamma=-0.4
$$

At the first step, let us determine the observer gain $K$ with the proposed iterative algorithm. The obtained gain $K$ and attenuation $\mu$ for a pole assignment in a disk centred at $(0.3,0)$ with radius 0.2 are :

$$
K=\left(\begin{array}{c}
0.1467 \\
4.6498 \\
-7.7861 \\
4.4794
\end{array}\right) \quad \mu=4.78
$$

The second step consists of finding the UI class for an exact decoupling. Let us recall that the state decoupling condition towards the UI with an exact estimation of the UI is given by (24). In this example, it corresponds to an UI composed of a linear combination of six exponential functions : two roots correspond to $\Lambda$ and $\lambda$, the others are two complex conjugate and two real values given by :

$$
\begin{gathered}
\lambda_{1}=\Lambda=1 ; \lambda_{2}=\lambda=0.7 ; \lambda_{3}=0.36 ; \lambda_{4}=0.29 \\
\lambda_{5,6}=0.43 \pm 0.02 i
\end{gathered}
$$

Then, the class of UI for an exact decoupling is given by :

$$
\eta_{k}=A_{1} \lambda_{1}^{k}+A_{2} \lambda_{2}^{k}+A_{3} \lambda_{3}^{k}+A_{4} \lambda_{4}^{k}+A_{5} a^{k} \cos (\phi k+\psi)
$$

with :

$a=\sqrt{\operatorname{Re}\left(\lambda_{5}\right)^{2}+\operatorname{Im}\left(\lambda_{5}\right)^{2}}$ and $\cos (\phi)=\frac{\operatorname{Re}\left(\lambda_{5}\right)^{2}}{2 \sqrt{\operatorname{Re}\left(\lambda_{5}\right)^{2}+\operatorname{Im}\left(\lambda_{5}\right)^{2}}}$

The UI is defined by :

$$
\begin{aligned}
& \eta_{k}=0.1-0.2(0.7)^{k}+0.4(0.36)^{k}+ \\
& 0.2(0.29)^{k}-0.5(0.93)^{k} \cos (2.05 k)
\end{aligned}
$$

Finally, the considered UI $\eta_{k}$ can be written as $\eta_{k}=\eta_{k}^{d}+\eta_{k}^{a}$ with $\eta_{k}^{d}$ corresponds to the UI for exact decoupling and $\eta_{k}^{a}$ the approximation error. The following figures are obtained for the initial conditions $x_{0}=\left(\begin{array}{llll}0.5 & 0.1 & 0.2 & -0.1\end{array}\right)^{T}$ and $\hat{x}_{0}=\left(\begin{array}{llll}-0.5 & 0.5 & -0.4 & 0.2\end{array}\right)^{T}$. Fig.1 shows the system inputs. Fig. 2 represents the UIs (for the exact $\eta_{k}=\eta_{k}^{d}$ and partial decoupling cases $\eta_{k}=\eta_{k}^{d}+\eta_{k}^{a}$ ) and their estimates and Fig 3. represents the state system and their estimate for both situations of exact and partial decoupling. In both situations, the state estimate is satisfactory.

In the case where a noise $r$ affects the output measurment, we considere the new output defined as :

$$
y_{k}=C x_{k}+e \eta_{k-1}+W r_{k}
$$

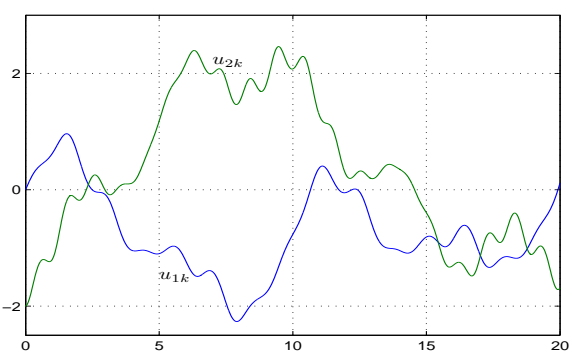

Fig. 1. System inputs
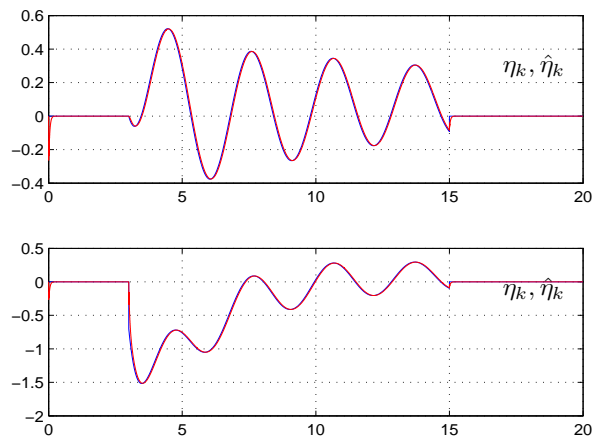

Fig. 2. UI and its estimate :exact decoupling (top) $\mathscr{L}_{2}$-attenuation (bottom)
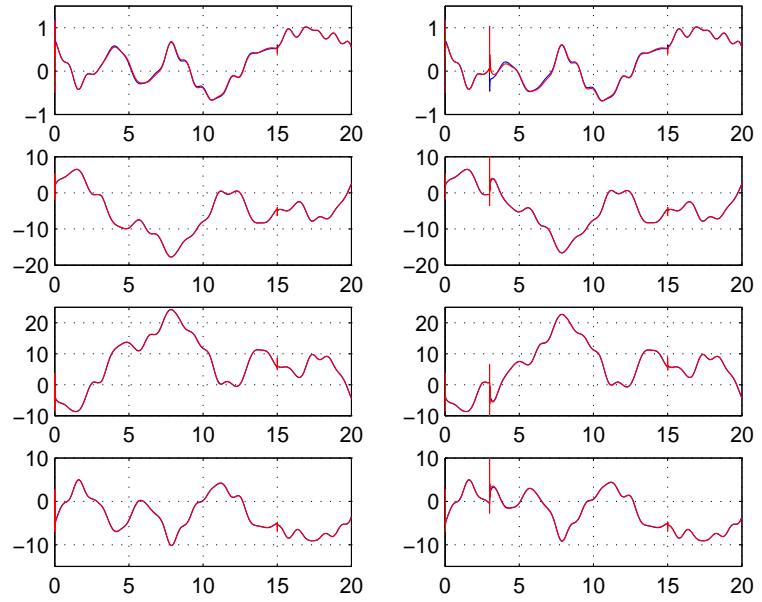

Fig. 3. System states and their estimates : exact decoupling (left) $\mathscr{L}_{2}$ attenuation (right)

Our goal is to attenuate the influence of the UI and the measurment noise on the state estimation error. To minimize their effect, we filter the output reconstruction error $\tilde{y}$ with a Low Pass Filter with a constant time of $(1-\alpha)$ :

$$
\tilde{y}_{f, k}=\alpha \tilde{y}_{k}+(1-\alpha) \tilde{y}_{f, k-1}
$$

By replacing this equation in the observer equations, we get :

$$
\left\{\begin{aligned}
\hat{x}_{k+1} & =A \hat{x}_{k}+B u_{k}+D \hat{\eta}_{k-1}+K \tilde{y}_{f, k} \\
z_{k+1} & =\Gamma \tilde{y}_{f, k}+\lambda z_{k}
\end{aligned}\right.
$$


The different output and UI estimate (with and without filter) obtained for a weightning matrix of $10 \%$ and a coefficient filter $\alpha=0.25$ are presented in the figures 4 and 5 :
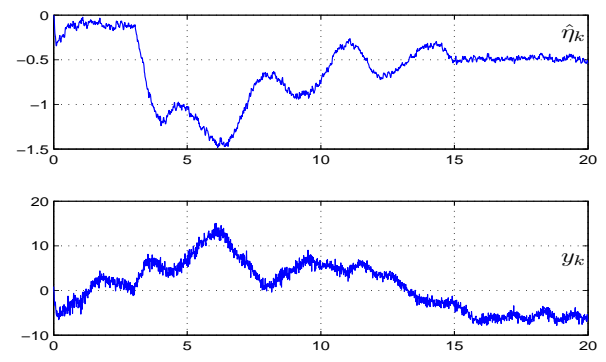

Fig. 4. UI estimate and system output without filter
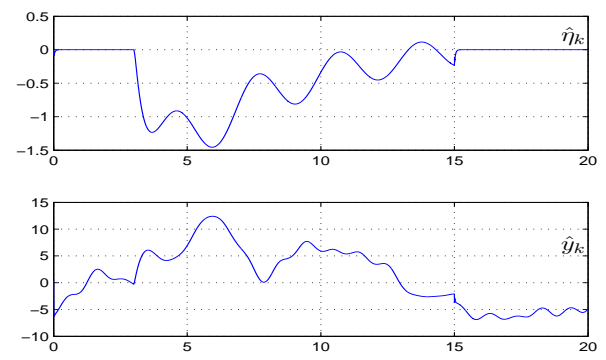

Fig. 5. UI estimate and system output with filter

The simulation results of the UI estimation obtained by the proposed observer are displayed on the previous figures. Solving the LMIs (37) may cause slow dynamics of the observer, so an eigenvalue assignment in a $D$-region allows to increase the performances of the observer. Also, in case of measurment noise, a filter is added at the output to improve the performances.

\section{Conclusion And Perspectives}

This paper addresses new method to design observers with unknown inputs. The proposed approach is based on a decoupling and estimation procedure such that we decouple the state system towards exponential UI without any rank constraints on the system's matrix. The main result is about the way to find the UI class ensuring an exact decoupling. The proposed work can be extended to the nonlinear case, in particular, systems with Takagi-Sugeno representation.

\section{REFERENCES}

[1] A. Akhenak, D. Maquin, and J. Ragot, "Process input estimation with a multimodel. Application to communication", Proceedings of the $13^{\text {th }}$ Mediterranean Conference on Control and Automation, Limassol, Cyprus, June 27-29, 2005.

[2] G. Basile and J. Marro, On the observability of linear, time-invariant systems with unknown inputs, Journal of optimization Theory and applications, vol. 3, 1969, pp 410-415.

[3] S. Boyd, L. El Ghaoui, E. Feron and V. Balakrishnan, Matrix Inequalities in System and Control Theory, SIAM ed; 1994.

[4] S-K Chang, Design of General Structured Observers for Linear Systems with Unknown Inputs, Journal Franklin Institute, vol. 334 (2), 1997, pp 213-232.
[5] JM. Chen and C. Chen, Unknown input observer for linear nonminimum phase systems, Journal of the Franklin Institute, vol. 347, 2010, pp 577-588.

[6] M. Chilali and P. Gahinet, $H_{\text {infty }}$ design with pole placement constraints : an LMI approach, IEEE Transactions on Automatic Control, vol. 29 (7), 2010, pp 603-614.

[7] M. Darouach, M. Zasadzinski and S. Xu, Full-order observers for linear systems with unknown inputs, IEEE Transactions on Automatic Control, vol. 39 (3), 1994, pp 606-609.

[8] M.C. de Oliveira, J. Bernussou and J.C. Geromel, A new discrete-time robust stability condition, Systems and Control Letters, vol. 37, 1999, pp 261-265.

[9] R. Guidorzi and G. Marro, On Wonham stabilizability condition in the synthesis of observers for unknown input systems, IEEE Transactions on Automatic Control, vol. 16, 1971, pp 499-500.

[10] H. Hammouri and Z. Tmar, Unknown input observer for state affine systems : A necessary and sufficient condition, Automatica, vol. 46, 2010, pp 271-278.

[11] G. Hostetter and J. Meditch, Observing systems with unmeasurable inputs, IEEE Transactions on Automatic Control, vol. 18, 1973, pp 307-308.

[12] M. Hou and P. Muller, Design of observers for linear systems with unknown inputs, IEEE Transactions on Automatic Control, vol. 37 (6), 1992, pp 871-875.

[13] D. Ichalal, B. Marx, J. Ragot and D. Maquin, "State and unknown input estimation for nonlinear systems described by Takagi-Sugeno models with unmeasurable premise variables", Proceedings of the $17^{\text {th }}$ Mediterranean Conference on Control and Automation, MED09, Thessaloniki, Greece, 2009.

[14] D. Ichalal, B. Marx, J. Ragot and D. Maquin, "Simultaneous state and unknown inputs estimation with PI and PMI observers for Takagi Sugeno model with unmeasurable premise variables", Proceedings of the $17^{\text {th }}$ Mediterranean Conference on Control and Automation, MED09, Thessaloniki, Greece, 2009.

[15] T. Iwasaki and R.E. Skelton, The XY-centring algorithm for the dual LMI problem : a new approach to fixed-order control design, International Journal of Control, vol. 62 (6), 1995, pp 1257-1272.

[16] C. Johnson, On observers for systems with unknown and inacessible inputs, IEEE Transactions on Automatic Control, vol. 21, 1975, pp 825- 831.

[17] A. Khedir, K. Benothman, D. Maquin and M. Benrejeb, "State and Sensor Faults Estimation via a Proportional Integral Observer", $6^{\text {th }}$ International Multi-conference on Systems signals and devices SSD'09, Djerba, Tunisia, March 23-26 2009.

[18] Zs. Lendek, J. Lauber, T.M. Guerra, R. Babuska and B. De Schutter, Adaptive observers for TS fuzzy systems with unknown polynomial inputs, Fuzzy Sets and Systems, vol. 16 (1), 2010, pp 2043- 2065.

[19] Y. Losser, Ajustement de lois de commande : Application en aéronautique, $\mathrm{PhD}$ Thesis, Ecole Nationale Supérieure de l'Aéronautique et de l'Espace, Mars 2006.

[20] B. Marx, D. Koenig and J. Ragot, Design of observers for TakagiSugeno descriptor systems with unknown inputs and application to fault diagnosis, IET Control Theory and Applications, vol. 1 (5), 2005 , pp 1487- 1495 .

[21] D. Peaucelle, D. Arzelier, O. Bachelier and J. Bernussou, A new robust D-stability condition for real convex polytopic uncertainty, Systems and Control Letters, vol. 40, 2000, pp 21-30.

[22] M.A. Rotea and T. Iwasaki, "An alternative to the D-K iteration", Proceedings of the American Control Conference, Baltimore, Maryland, USA, June 1994, pp. 53-57.

[23] A. Shumsky, "Algebraic Approach to the Problem of Fault Accommodation in Nonlinear Systems", Proceedings of the $17^{\text {th }}$ World Congress The International Federation of Automatic Control, Seoul, Korea, July 6-11 2008.

[24] Y. Yamada and S. Hara, An LMI approach to local optimization for constantly scaled $H_{\infty}$ control problems, International Journal of Control, vol. 67 (2), 1987, pp 233-250.

[25] F. Yang and R. Wilde, Observers for linear systems with unknown inputs, IEEE Transactions on Automatic Control, vol. 31 (7), 1988, pp 677-681.

[26] K. Zhou and P.P. Khargonekar, Robust stabilization of linear systems with norm-bounded time-varying uncertainty, Systems and Control Letters, vol. 10 (1), 1988, pp 17-20. 